\title{
Rosuvastatin Reduces Plasma Small Dense LDL-Cholesterol Predominantly in Non-Diabetic Hypercholesterolemic Patients
}

\author{
Gen Yoshino ${ }^{1}$, Saburo Nakano ${ }^{1}$, Tomoko Matsumoto ${ }^{1}$, Eiichi Murakami ${ }^{2}$, Toshisuke Morita ${ }^{3}$, \\ Koji Kuboki ${ }^{1}$ \\ ${ }^{1}$ Division of Diabetes, Metabolism and Endocrinology, Department of Internal Medicine, School of Medicine, Toho University, To- \\ kyo, Japan; ${ }^{2}$ Clinical Laboratory, Medical Center Omori Hospital, Toho University, Tokyo, Japan; ${ }^{3}$ Department of Laboratory Medi- \\ cine, School of Medicine, Toho University, Tokyo, Japan. \\ Email: yoshino@med.toho-u.ac.jp
}

Received October $4^{\text {th }}, 2011$; revised November $13^{\text {th }}, 2011$; accepted December $5^{\text {th }}, 2011$

\begin{abstract}
Aims: Small dense LDL (sdLDL) cholesterol is considered a cardiovascular risk. Our purpose in this study was to evaluate the efficacy of rosuvastatin in reducing sdLDL and large buoyant LDL (lbLDL-C) in hypercholesterolemia. Methods: Fifty-six patients with a mean baseline LDL-cholesterol (LDL-C) concentration of $173.9 \pm 40.5 \mathrm{mg} / \mathrm{dL}$ were treated with rosuvastatin $2.5 \mathrm{mg}$ /day for 12 weeks. LDL-C, sdLDL-C, and apolipoprotein (apo) B were assessed and l lbLDL-C was calculated (LDL-C minus sdLDL-C). Results: After 12-week treatment with rosuvastatin $2.5 \mathrm{mg}$, sdLDL-C and lbLDL-C were significantly reduced from $62.1 \pm 23.8 \mathrm{mg} / \mathrm{dL}$ to $34.0 \pm 13.4 \mathrm{mg} / \mathrm{dL}, \mathrm{p}<0.001$ and 112.7 $\pm 34.9 \mathrm{mg} / \mathrm{dL}$ to $77.2 \pm 29.2 \mathrm{mg} / \mathrm{dL}, \mathrm{p}<0.001$ respectively, and sdLDL-C/lbLDL-C ratio and apo $\mathrm{B}$ also decreased significantly, from $0.36 \pm 0.02$ to $0.32 \pm 0.02$, p $<0.005$ and $134.2 \pm 4.3$ to $93.6 \pm 3.5 \mathrm{mg} / \mathrm{dl}, \mathrm{p}<0.001$, respectively. In diabetic subjects there was significant correlation between percent reductions in the plasma triglyceride and sdLDL-C/ lbLDL-C ratio $(r=0.58, \mathrm{p}<0.005)$, but not between the percentage decrease in plasma triglyceride and sdLDL-C. Conclusions: Treatment with rosuvastatin is associated with significant reduction in sdLDL, lbLDL and sdLDL/lbLDL ratio.
\end{abstract}

Keywords: Rosuvastatin; Small Dense LDL-Cholesterol (sdLDL); Large Buoyant LDL-C (lbLDL-C); Apolipoprotein (apo) B; Small Dense LDL-Cholesterol (sdLDL)/Large Buoyant LDL-C (lbLDL-C) Ratio

\section{Introduction}

Increased plasma LDL-cholesterol (LDL-C) level is one of the most important risk factors for coronary artery disease (CAD) [1,2]. Plasma LDL comprises multiple discrete subclasses, differing in size and density. In particular, small dense LDL (sdLDL) has been demonstrated to be a new risk factor for the development of CAD [3-5]. Some studies have demonstrated that several HMG-CoA reductase inhibitors (statins) reduce not only LDL-C but also sdLDL [6-8]. However, it is a matter of debate whether statins reduce sdLDL or lbLDL predominantly. Diabetes mellitus is an independent risk factor for CAD [9]. It is known that sdLDL is dominant in type 2 diabetic patients $[10,11]$. Therefore, it is possible that the effect of statins on LDL size is modified by the presence of type 2 diabetes. Thus, the present study was conducted in order to examine whether rosuvastatin reduces sdLDL or lbLDL predominantly using a new sdLDL-C assay system [12].

\section{Patients and Methods}

Fifty-six patients with hypercholesterolemia (19 males and 37 females, including 24 type 2 diabetics) at the Division of Diabetes, Metabolism and Endocrinology, Omori Hospital Medical Center who had been receiving dietary treatment for dyslipidemia and/or type 2 diabetes mellitus were randomly enrolled in this study. All study protocols and procedures were approved by the Ethics Committee of Toho University Medical Center Omori Hospital. The study objectives and intended treatment were explained to 57 prospective study patients individually; 56 patients completed the study and 1 patient discontinued the treatment. Written informed consent was obtained from all 57 participants. Patients were excluded if they had any of the following conditions: 1) decreased renal function as defined by serum creatinine levels above 
$1.2 \mathrm{mg} / \mathrm{dl}$; 2) liver disease (serum aminotransferase above 3 times the upper limit of normal range (10 - 35 IU/l); 3) hyperthyroidism with free T4 above $1.0 \mathrm{mg} / \mathrm{dl}$; 4) pregnancy; 5) lipid-lowering therapy with fibrates, nicotinic acid, cholestimide, ezetimibe, or omega-3 fatty acids within at least 8 weeks before rosuvastatin administration. Also, diabetic patients under pioglitazone treatment were excluded. After a six-week dietary leading period with the NCEP-step-1 diet, eligible patients were prescribed rosuvastatin $2.5 \mathrm{mg} /$ day. Patients were advised not to change their instructed dietary habit during the study period. Before and 12 weeks after treatment with $2.5 \mathrm{mg}$ of rosuvastatin, plasma lipids, including LDL-C, sdLDL-C, and apolipoprotein (apo) B, were measured. LbLDL-C was calculated by subtracting sdLDL-C from LDL-C. LbLDL-C values estimated by this method were reported to be well correlated with the values determined by ultracentrifugation $(\mathrm{r}=0.858$, $\mathrm{p}<$ 0.0001 ) [12]. LDL- C and sdLDL-C was measured by Okada et al. [13] and by Hirano et al. [12], respectively. Apo B was measured by immunoturbidometry (Daiichi Chemicals Co., Tokyo, Japan).

All values were expressed as mean $\pm \mathrm{SD}$, but mean \pm SE was also employed for the comparison data before and after rosuvastatin treatment. Paired t test was employed for comparison of the data before and after rosuvastatin treatment. The comparison of changes in plasma lipids after rosuvastatin treatment between diabetic and non-diabetic patients was performed by analysis of covariance (ANCOVA) taking the baseline values of these parameters as a covariate. Simple regression analysis was performed to examine the association between percent reductions in plasma triglyceride and sdLDL-C or sdLDL-C/lbLDL-C ratio in both diabetic and non-diabetic subjects. A significant difference was defined as $\mathrm{p}$ $<0.05$.

\section{Results}

The clinical characteristics of the 56 patients who completed this study are shown in Tables $\mathbf{1}$ and $\mathbf{2}$. The study subjects consisted of 19 males and 37 females, with a mean age of 57.6 years ( 36 - 78 years). The mean BMI was $26.1 \pm 6.8$. Twenty-three diabetic patients and 13 hypertensive patients were included. Plasma TC and LDL-C levels in all patients significantly decreased from $260.3 \pm 42.5$ and $173.9 \pm 40.5 \mathrm{mg} / \mathrm{dL}$, to $192.1 \pm 30.1$ $\mathrm{mg} / \mathrm{dL}$ and $109.6 \pm 28.4 \mathrm{mg} / \mathrm{dL}$, respectively, after the 12-week rosuvastatin treatment $(\mathrm{p}<0.001)$ (Figure 1). Plasma TG levels also decreased, from $155.5 \pm 88.4$ $\mathrm{mg} / \mathrm{dL}$ to $136.6 \pm 42.5 \mathrm{mg} / \mathrm{dL}$ ( $\mathrm{p}<0.05$ ) (Figure 2). Both sdLDL-C and lbLDL-C decreased significantly from $62.1 \pm 23.8 \mathrm{mg}$ and $112.7 \pm 34.9 \mathrm{mg} / \mathrm{dL}$ to $34.0 \pm 13.4$
Table 1. Characteristics of all study subjects.

\begin{tabular}{ccc}
\hline \multicolumn{3}{c}{ Number of patients } \\
\hline Sex (male: female) & & $19: 37$ \\
Age (years) & 56 & $58.6 \pm 14.0$ \\
TC (mg/dL) & 56 & $260.3 \pm 42.5$ \\
TG (mg/dL) & 56 & $156.5 \pm 88.4$ \\
LDL-C (mg/dL) & 56 & $173.9 \pm 40.5$ \\
HDL-C (mg/dL) & 56 & $59.5 \pm 16.3$ \\
HbA1c (\%) & 23 & $7.2 \pm 1.5$ \\
BMI & 56 & $24.8 \pm 6.0$ \\
SBP (mmHg) & 56 & $127.4 \pm 12.6$ \\
DBP (mmHg) & 56 & $73.5 \pm 11.5$ \\
FBS (mg/dL) & 56 & $123.1 \pm 36.8$ \\
\hline
\end{tabular}

TC: total cholesterol, TG: triglyceride, LDL-C: low density lipoprotein cholesterol, HDL-C: high density lipoprotein cholesterol, HbA1c: hemoglobin A1c, BMI: body mass index, SBP: systolic blood pressure, DBP: diastolic blood pressure; Data was expressed as mean \pm SD and the data in the parenthesis indicate the normal range; ${ }^{*} \mathrm{HbA1c}$ value was indicated as Japan Diabetes Society (JDS) scale.

Table 2. Characteristics of non-diabetic and diabetic subjects.

\begin{tabular}{ccc}
\hline & Diabetic patients & Non-diabetic patients \\
\hline Sex (male/female) & $11 / 12$ & $8 / 25$ \\
Age (years) & $57.8 \pm 18.8$ & $59.6 \pm 19.5$ \\
HbA1c (\%) & $7.22 \pm 1.5$ & $24.0 \pm 9.0$ \\
BMI & $26.1 \pm 6.8$ & $24.0 \pm 9.0$ \\
SBP (mmHg) & $127.8 \pm 18.0$ & $126.8 \pm 17.3$ \\
DBP (mmHg) & $74.9 \pm 16.5$ & $73.6 \pm 12.8$ \\
FBS (mg/dL) & $150.1 \pm 52.5$ & $99.8 \pm 20.3$ \\
Concomitant medications (n) & & \\
Rosuvastatin alone & 9 & 29 \\
ARB & 10 & 1 \\
CCB & 5 & 2 \\
ACE-I & 0 & 0 \\
SU & 10 & 0 \\
BG & 9 & 0 \\
$\alpha$-GI & 2 & 1 \\
\hline
\end{tabular}

Data was expressed as mean \pm SD; HbA1c: hemoglobin A1c, BMI: body mass index, SBP: systolic blood pressure, DBP: diastolic blood pressure, FBS: Fasting blood glucose, ARB: angiotensin II receptor blockers, CCB: calcium channel blockers, ACR-I: angiotensin converting enzyme-inhibitors, SU: sulphonylureas, BG: biguanides, $\alpha$-GI: $\alpha$-glucosidase inhibitors. 


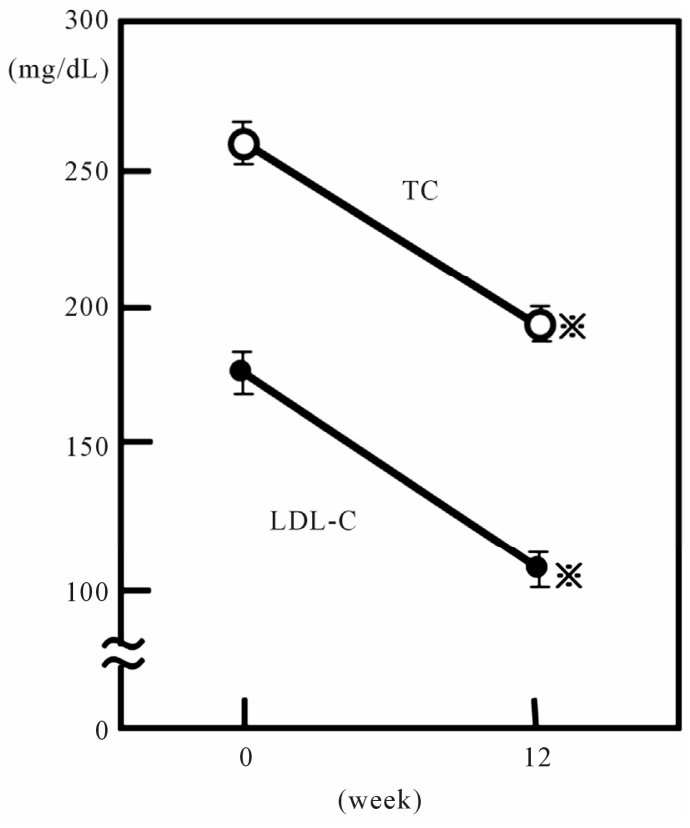

Figure 1. Effect of $2.5 \mathrm{mg}$ daily of rosuvastatin on plasma total-cholesterol and LDL-cholesterol in all 56 hypercholesterolemic subjects. Open and closed circles represent the data of total-cholesterol (TC) and LDL-cholesterol (LDL-C), respectively. Vertical bars represent mean $\pm \mathrm{SE}$. *: significantly different from 0 week value $(p<0.001$, by paired-t test).

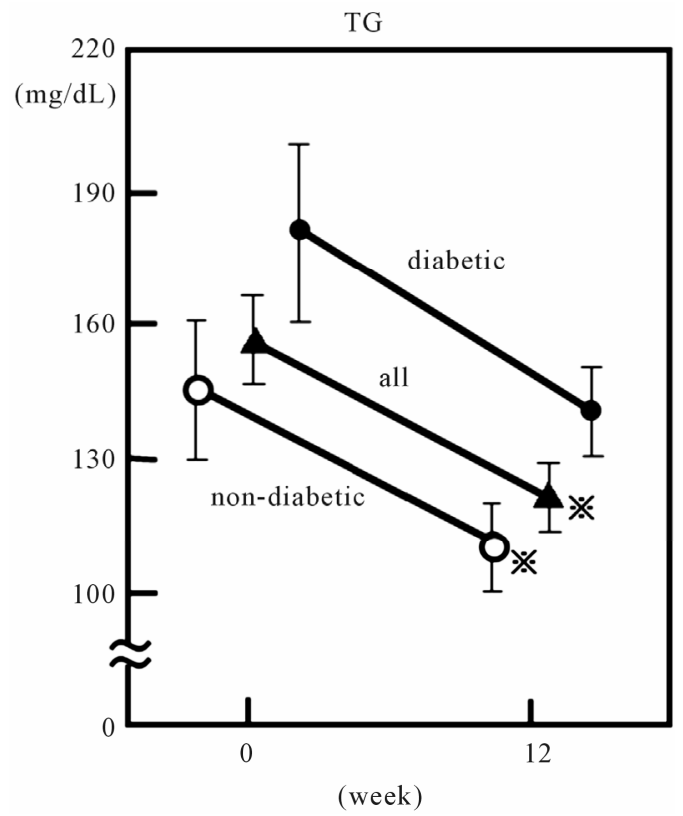

Figure 2. Effect of $2.5 \mathrm{mg}$ daily of rosuvastatin on plasma triglyceride (TG) in 56 hypercholesterolemic subjects. Open circles, closed circles and triangles represent the data of non-diabetic $(n=33)$, diabetic $(n=23)$ and all subjects, respectively. Vertical bars represent mean $\pm \mathrm{SE}$. *: significantly different from 0 week value $(p<0.001$, by paired-t test). $\mathrm{mg} / \mathrm{dL}$ and $77.2 \pm 29.2 \mathrm{mg} / \mathrm{dL}$ respectively after the 12 week rosuvastatin treatment $(\mathrm{p}<0.001$ and $<0.001$, respectively) (Figure 3) and sdLDL-C/lbLDL-C ratio also decreased significantly from $0.36 \pm 0.02$ to $0.32 \pm 0.02$ (p < 0.005) (Figure 4).

Plasma apo B also decreased, from $134.2 \pm 4.3$ to 93.6 $\pm 3.5 \mathrm{mg} / \mathrm{dl}(\mathrm{p}<0.001)$. Although HDL-C level did not show significant change, the LDL-C/HDL-C ratio significantly decreased from $3.10 \pm 0.14$ to $1.89 \pm 0.09$ (p < 0.001 ) after rosuvastatin treatment. When the patients were divided into two groups according to presence or absence of type 2 diabetes, both sdLDL-C and lbLDL-C decreased significantly after treatment in both groups. However, a significant decrease in plasma TG and sdLDL-C/lbLDL-C ratio was found only in non-diabetic patients (Figures 2, 4 and Table 3).

Thereafter ANCOVA was conducted. The independent variable was presence or absence of type 2 diabetes. The dependent variable were the plasma lipid levels including TC, TG LDL-C, sdLDL-C, lbLDL-C, sdLDL-C/lbLDL$\mathrm{C}$ ratio and HDL-C, after rosuvastatin treatment and the covariate was the same plasma lipid levels before treatment. The ANCOVA was significant only when the plasma TC and lbLDL-C were applied as dependent variable and covariate $(\mathrm{p}<005)$. The association between percent reductions in the plasma triglyceride and sdLDL-C or sdLDL-C/lbLDL-C ratio was examined in diabetic and non-diabetic subjects. In diabetic subjects there was significant correlation between percent reductions in plasma triglyceride and sdLDL-C/lbLDL-C ratio $(r=0.58, p<0.005)$ but not between percent reductions of plasma triglyceride and sdLDL-C. In addition, there was no significant correlation between percent reduction of plasma triglyceride and sdLDL-C/lbLDL-C ratio, or plasma triglyceride and sdLDL-C in non-diabetic subjects.

\section{Discussion}

It is well known that there are multiple density and size subclasses in plasma LDL fraction; Krauss and Burke [14] have demonstrated that two distinct LDL subclass phenotypes can be distinguished on the basis of the LDL particle distribution separated by gradient gel electrophoresis (GEG). Pattern A consists of a major peak greater than $25.5 \mathrm{~nm}$, whereas in pattern $B$ this is less than $25.5 \mathrm{~nm}$. An investigation of LDL subfractions in normotriglyceridemic men revealed that sd LDL can be a cardiovascular risk factor in subjects without apparent hyperlipidemia [15]. LDL composition was examined in type 2 diabetic patients. The decrease in cholesterolloading in LDL and increase in the number of LDL particles was marked in normolipidemic diabetic patients, 


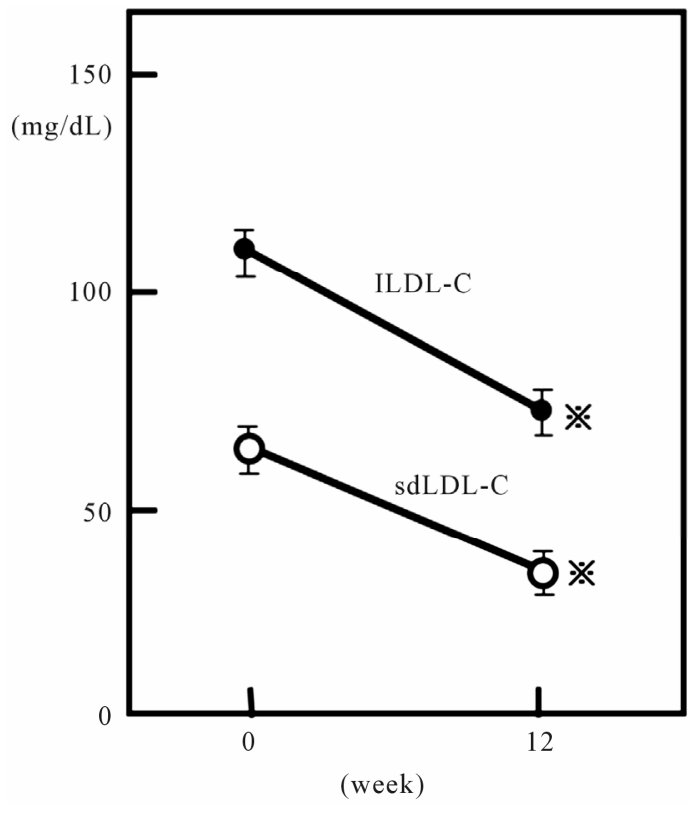

Figure 3. Effect of $2.5 \mathrm{mg}$ daily of rosuvastatin on plasma large LDL-cholesterol and sdLDL-cholesterol in all 48 hypercholesterolemic subjects. Closed and open circles represent the data of large buoyant LDL-cholesterol (lbLDL-C) and small dense LDL-cholesterol (sdLDL-C), respectively. Vertical bars represent mean $\pm \mathrm{SE}$. *: significantly different from 0 week value ( $p<0.001$, by paired-t test).

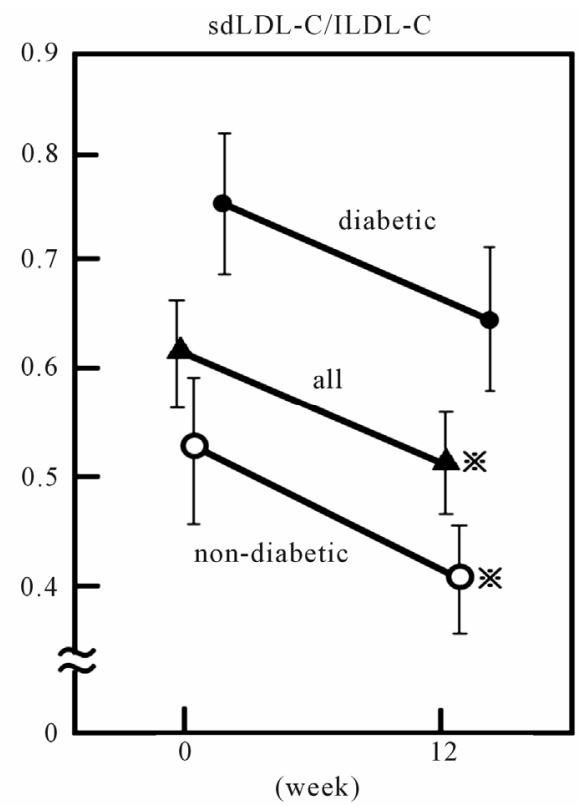

Figure 4. Effect of $2.5 \mathrm{mg}$ daily of rosuvastatin on plasma small dense LDL-cholesterol/large LDL-cholesterol (sdLDLC/lbLDL-C) ratio in 56 hypercholesterolemic subjects. Open circles, closed circles and triangles represent the data of non-diabetic $(n=33)$, diabetic $(n=23)$ and all subjects, respectively. Vertical bars represent mean $\pm S E$. *: significantly different from 0 week value $(p<0.001$, by paired-t test).
Table 3. Changes in plasma lipid levels of diabetic and nondiabetic patients before and after rosuvastatin treatment.

\begin{tabular}{ccccc}
\hline \multirow{2}{*}{$\begin{array}{c}\text { Number of } \\
\text { patients }\end{array}$} & \multicolumn{2}{c}{ Diabetic patients } & \multicolumn{2}{c}{ Non-diabetic patients } \\
\cline { 2 - 5 } & \multicolumn{2}{c}{23} & \multicolumn{2}{c}{33} \\
\hline $\begin{array}{c}\text { rosuvastatin } \\
\text { treatment }\end{array}$ & before & after & before & after \\
\hline TC & $251 \pm 9^{* *}$ & $179 \pm 6$ & $268 \pm 8^{* *}$ & $202 \pm 6$ \\
TG & $178 \pm 22$ & $139 \pm 11$ & $145 \pm 15^{*}$ & $114 \pm 11$ \\
LDL-C & $173 \pm 21^{* *}$ & $102 \pm 6$ & $176 \pm 9^{* *}$ & $115 \pm 6$ \\
HDL-C & $50 \pm 2$ & $54 \pm 3$ & $64 \pm 3$ & $66 \pm 3$ \\
LDL-C/HDL-C & $3.5 \pm 0.2^{* *}$ & $2.1 \pm 0.2$ & $2.9 \pm 0.2^{* *}$ & $1.8 \pm 0.1$ \\
sdLDL-C & $70 \pm 5^{* *}$ & $38 \pm 3$ & $57 \pm 4^{* *}$ & $31 \pm 2$ \\
\hline
\end{tabular}

DM: type 2 diabetics mellitus, n:number of subjects, TC: total-cholesterol; TG: triglyceride, LDL-C: low-density lipoprotein cholesterol; HDL-C: high-density lipoprotein cholesterol, sdLDL-C: small dense LDL-cholesterol Date was expressed as mean $\pm \mathrm{SE}$; There are significant differences between the data before and after rosuvastatin treatment by paired $t$-test $\left({ }^{*}: \mathrm{p}<0.05\right.$, : $\mathrm{p}<0.001)$.

indicating the predominance of sdLDL in subjects with type 2 diabetes [16]. Furthermore, acute myocardial infarction survivors with type 2 diabetes demonstrated "pattern B" [17,18].

LDL size is usually measured by gradient gel electrophoresis using polyacrylamide gel [14]. This standard assay procedure takes a long time and is costly. However, Hirano et al. have developed a simple and rapid assay system for the measurement of sdLDL-cholesterol concentration using heparin-magnesium precipitation and direct LDL assay [12]. This assay system enabled us to measure absolute value of sdLDL-C and large LDL-C separately. In addition, there was very good correlation between lbLDL-C value calculated by this system and that measured after ultracentrifugation [12].

In particular, sdLDL has been demonstrated to be a new risk factor for the development of CAD in Western countries as well as in Japan [3-5,17]. Some studies have demonstrated that several HMG-CoA reductase inhibitors (statins) reduce not only LDL-C, but also sdLDL [6-8,19-22]. However, it is a matter of debate whether statins reduce sd LDL or lbLDL predominantly. Moreover, diabetes mellitus is an independent risk factor for CAD [9]. It is known that sdLDL is dominant in type 2 diabetic patients $[10,11]$. Therefore, it is possible that the effect of statins on LDL size is modified by the presence of type 2 diabetes.

Rosuvastatin has come into widespread clinical use because it has been reported to exhibit a stronger LDL-C lowering effect and a higher rate of achieving therapeutic goals than atorvastatin [23]. Thus, the present study was conducted in order to examine whether rosuvastatin re- 
duces sb LDL or lbLDL predominantly using a newly developed sdLDL-C assay system [12].

In this study, administration of rosuvastatin resulted in a decrease in sdLDL-C as well as apo B and lbLDL-C. As plasma apo B indicates the LDL particle number in plasma [24], it is plausible that rosuvastatin not only reduced LDL particle number but also suppressed population of sdLDL and lbLDL fraction. Furthermore, rosuvastatin reduced the sdLDL-C per lbLDL-C ratio. However, when the subjects were divided into two groups according to presence or absence of type 2 diabetes, significant reduction of sdLDL-C/lbLDL-C ratio was found only in the non-diabetic subjects. This suggests that rosuvastatin suppressed smaller-sized LDL production predominantly in subjects without type 2 diabetes.

The proposed mechanisms for generation of smaller-sized LDL in diabetic subjects are as follows $[25,26]$. There is substantial evidence that type 2 diabetes and/or insulin resistance may increase free fatty acid release into the circulation from adipocytes and elevated plasma glucose. The liver may increase triglyceride production utilizing these increased free fatty acids and glucose from the plasma as substrates. As a consequence of increased hepatic triglyceride synthesis, there may be increased production of triglyceride-rich (large-sized) very lowdensity lipoprotein (VLDL) in the liver. This triglyceride-rich VLDL can be a precursor of sdLDL. Triglyceride-rich VLDLs are converted to triglyceride-enriched LDLs, which are favored substrates for hepatic lipase, and transformed into smaller-sized LDL by lipase-mediated triglyceride hydrolysis.

It is a matter of debate how rosuvastatin reduced the population of sd LDL predominantly in non-diabetic subjects. Plasma triglyceride was suppressed significantly in non-diabetic subjects in this study, and it is possible that reduction of plasma sdLDL-C after rosuvastatin treatment may have been caused by suppression of hepatic triglyceride production by rosuvastatin in this patient group.

It is of interest to know that when the patients were divided into two groups according to presence or absence of type 2 diabetes; the significant decrease in the sdLDLC/lbLDL-C ratio was found only in non-diabetic patients. It is well-known that the baseline triglyceride level is the most important independent variable associated with the triglyceride-lowering effect of rosuvastatin [20-22]. Yet, in this study rosuvastatin was not able to decrease plasma triglyceride levels in diabetic patients who exhibit relatively higher baseline triglyceride levels. One of the reasons for this discrepancy may be due to the much low dose of rosuvastatin in our study. Hypertriglyceridemia in diabetic subjects may be due to increased production of hepatic triglyceride and to impaired lipoprotein lipase activity [10]. It is possible that low doses of rosuvastatin can decrease production of hepatic triglyceride, but cannot increase lipoprotein lipase activity in diabetic subjects, i.e., the low dose of rosuvastatin treatment cannot fully normalize impaired triglyceride metabolism and therefore is not able to suppress smaller-sized LDL production predominantly in these patients.

We compared the changes in lipid parameters after rosuvastatin administration between diabetic and non-diabetic patients employing ANCOVA and taking the baseline values of the same plasma lipid levels as a covariate. However, a significant difference was found in the effect of rosuvastatin only on plasma TC and lbLDL$C$ between the diabetic and non-diabetic group using ANCOVA. This indicates that the differences in the baseline values of plasma triglyceride were not able to influence the results analyzed by the paired-t test.

In diabetic subjects there was significant correlation between percent reduction in plasma triglyceride and the sdLDL-C/lbLDL-C ratio, but not between plasma triglyceride and sdLDL-C. On the other hand, there was no significant correlation between plasma triglyceride and the sdLDL-C/lbLDL-C ratio or between plasma triglyceride and sdLDL-C in non-diabetic subjects. These results suggest that the LDL peak size was influenced by the plasma triglyceride metabolism in diabetic subjects, but this influence was weak in non-diabetic subjects because their base line plasma triglyceride was much lower than that of the diabetic subjects. Thus, though it is possible that rosuvastatin reduces plasma sdLDL mainly through improving triglyceride metabolism there may be other mechanisms for plasma sdLDL reduction.

This was a small, open-label, prospective study, and the observation period was only 3 months. Thus, the longterm prognosis is not known. Therefore, further study is necessary to confirm the effect of rosuvastatin on LDL size distribution in diabetic and non-diabetic hypercholesterolemic patients.

In conclusion, rosuvastatin reduced plasma smallersized LDL and larger LDL, though the effect was markedly greater with the former. However, this effect was significant only in non-diabetic hypercholesterolemic patients.

\section{REFERENCES}

[1] S. M. Grundy, J. I. Cleeman, C. N. Merz, H. B. Brewer Jr., L. T. Clark, D. B. Hunninghake, R. C. Pasternak, S. C. Smith Jr. and N. J. Stone, "Coordinating Committee of the National Cholesterol Education Program, Implications of Recent Clinical Trials for the National Cholesterol Education Program Adult Treatment Panel III Guidelines," Journal of the American College of Cardiology, Vol. 44, No. 3, 2004, pp. 720-32. 
doi:10.1016/j.jacc.2004.07.001

[2] Lipid Research Clinics Program, "The Lipid Research Clinics Primary Prevention Trial Results. II, The Relationship of Reduction in Incidence of Coronary Heart Disease to Cholesterol Lowering," Journal of the American Medical Association (JAMA), Vol. 251, No. 3, 1984, pp. 365-374. doi:10.1001/jama.1984.03340270043026

[3] M. A. Austin, J. L. Breslow, C. H. Hennekens, J. E. Buring, W. C. Willett and R. M. Krauss, "Low-Density Lipoprotein Subclass Patterns and Risk of Myocardial Infarction," Journal of the American Medical Association (JAMA), Vol. 260, No. 13, 1988, pp. 1917-1921. doi:10.1001/jama.1988.03410130125037

[4] B. A. Griffin, D. J. Freeman, G. W. Tait, J. Thomson, M. J. Caslake, C. J. Packard and J. Shepherd, "Role of Plasma Triglyceride in the Regulation of Plasma Low Density Lipoprotein (LDL) Subfractions: Relative Contribution of Small Dense LDL to Coronary Heart Disease Risk,” Atherosclerosis, Vol. 106, No. 2, 1994, pp. 241253. doi:10.1016/0021-9150(94)90129-5

[5] T. Hirano, Y. Ito, S. Koba, M. Toyoda, A. Ikejiri, H. Saegusa, J. Yamazaki and G. Yoshino, "Clinical Significance of Small Dense Low-Density Lipoprotein Cholesterol Levels Determined by the Simple Precipitation Method," Arteriosclerosis, Thrombosis, and Vascular Biology, Vol. 24, No. 3, 2004, pp. 558-563. doi:10.1161/01.ATV.0000117179.92263.08

[6] T. Nozue, I. Michishita, Y. Ito and T. Hirano, "Effects of Statin on Small Dense Low Density-Lipoprotein Cholesterol and Remnant-Like Particle Cholesterol in Heterozygous Hypercholesterolemia," Journal of Atherosclerosis and Thrombosis, Vol. 15, No. 3, 2008, pp. 146153. doi:10.5551/jat.E552

[7] A. Tokuno, T. Hirano, T. Hayashi, Y. Mori, T. Yamamoto, M. Nagashima, Y. Shiraishi, Y. Ito and M. Adachi, "The Effects of Statin and Fibrate on Lowering Small Dense LDL-Cholesterol in Hyperlipidemic Patients with Type 2 Diabetes," Journal of Atherosclerosis and Thrombosis, Vol. 14, No. 3, 2007, pp. 128-132. doi:10.5551/jat.14.128

[8] A. Ikejiri, T. Hirano, S. Murayama, G. Yoshino, N. Gushiken, T. Hyodo, T. Taira and M. Adachi, "Effects of Atorvastatin on Triglyceride-Rich Lipoproteins, LowDensity Lipoprotein Subclass, and C-Reactive Protein in Hemodialysis Patients,” Metabolism, Vol. 53, No. 9, 2004, pp. 1113-1117. doi:10.1016/j.metabol.2004.01.011

[9] R. C. Turner, R. R. Holman, H. Millns, H. A. Neil, I. M. Stratton, S. E. Manley and D. R. Matthews, "Risk Factors for Coronary Artery Disease in Non-Insulin Dependent Diabetes Mellitus: UK Prospective Diabetes Study (UKPDS 23)," British Medical Journal (BMJ), Vol. 316, No. 7134, 1998, pp. 823-828. doi:10.1136/bmj.316.7134.823

[10] K. R. Feingold, C. Grunfeld, M. Pan, W. Doerrler and R. M. Krauss, "LDL Subclass Phenotypes and Triglyceride Metabolism in Non-Insulin-Dependent Diabetes," Arteriosclerosis, Thrombosis, Vol. 12, No. 12, 1992, pp. 14961502. doi:10.1161/01.ATV.12.12.1496
[11] T. Hirano, K. Oi, S. Sakai, K. Kashiwazaki, M. Adachi and G. Yoshino, "High Prevalence of Small LDL in Diabetic Nephropathy Is Not Directly Associated with Kidney Damage: A Possible Role of Postprandial Lipemia," Atherosclerosis, Vol. 141, No. 1, 1998, pp. 77-85. doi:10.1016/S0021-9150(98)00150-6

[12] T. Hirano, Y. Ito, H. Saegusa and G. Yoshino, “A Novel and Simple Method for Quantification of Small Dense Low-Density Lipoprotein,” Journal of Lipid Research, Vol. 44, No.11. 2003, pp. 2193-2201. doi:10.1194/jlr.D300007-JLR200

[13] M. Okada, H. Matsui, Y. Ito, A. Fujiwara and K. Inano, "Low-Density Lipoprotein Can Be Chemically Measured: A New Superior Method," Journal of Laboratory and Clinical Medicine, Vol. 132, No. 3, 1998, pp. 195-201. doi:10.1016/S0022-2143(98)90168-8

[14] R. M. Krauss and D. J. Burke, "Identification of Multiple Subclass of Plasma Low Density Lipoproteins in Normal Humans,” Journal of Lipid Research, Vol. 23, 1982, pp. 97-104.

[15] I. Rajiman, M. JKenall, R. Cramb, R. L. Holder, M. Salih and M. D. Gambage, "Investigation of Low Density Lipoprotein Subfractions as a Coronary Risk Factor in Normotriglyceridemic Men,” Atherosclerosis, Vol. 125, No. 2, 1996, pp. 231-242. doi:10.1016/0021-9150(96)05881-9

[16] M. Iwai, G. Yoshino, M. Matsushita, M. Morita, K. Matsuba, T. Kazumi and S. Baba, "Abnormal Lipoprotein Composition in Normolipidemic Diabetic Patients,” Diabetes Care, Vol. 13, No. 7, 1990, pp. 792-796. doi:10.2337/diacare.13.7.792

[17] G. Yoshino, T. Hirano and T. Kazumi, "Atherogenic Lipoproteins and Diabetes Melltus,” Journal of Diabetes and its Complications, Vol. 16, No. 1, 2002, pp. 29-34. doi:10.1016/S1056-8727(01)00199-4

[18] S. Koba, Y. Yokota, T. Hirano, Y. Ito, Y. Ban, F. Tsunoda, T. Sato, M. Shoji, H. Suzuki, E. Genshi, Y. Kobayashi and T. Katagiri, "Small LDL-Cholesterol Is Superior to LDL-Cholesterol for Determinin Severe Coronary Atherosclerosis," Journal of Atherosclerosis and Thrombosis, Vol. 15, No. 5, 2008, pp. 250-260. doi:10.5551/jat.E572

[19] G. Yoshino, T. Hirano and T. Kazumi, "Treatment of Small Dense LDL," Journal of Atherosclerosis and Thrombosis, Vol. 9, No. 6, 2002, pp. 266-275. doi:10.5551/jat.9.266

[20] M. J. Caslake, G. Stwart, S. P. Day, E. Daly, F. McTaggart, M. J. Chapman, P. Durrington, P. Laggner, M. Mackness, J. Pears and C. J. Packard, "PhenotypeDependent and -Independendent Actions of Rosuvastatin on Atherogenc Lipoprotein Subfractions in Hyperlipidaemia," Atherosclerosis, Vol. 171, No. 2, 2003, pp. 245253. doi:10.1016/j.atherosclerosis.2003.08.025

[21] M. S. Kostapanos, H. J. Milionis, T. D. Filippatos, E. S. Nakou, E. T. Baikaktari, A. D. Tselepis and M. S. Elisaf, "A 12-Weeks, Prospective, Open-Label Analysis of the Effect of Rosuvastatin on Triglyceride-Rich Lipoprotein Metabolism in Patients with Primary Dyslipidemia," Clinical Therapeutics, Vol. 29, No. 7, 2007, pp. 1403- 
1414. doi:10.1016/j.clinthera.2007.07.019

[22] M. S. Kostapanos, H. J. Milionis, K. G. Lagos, C. B. Rizos, A. D. Tselepis and M. S. Elisaf, "Baseline Triglyceride Levels and Insulin Sensitivity Are Major Determinants of the Increase of LDL Particle Size and Buoyancy Induced by Rosuvastatin Treatment in Patients with Primary Hyperlipidemia," European Journal of Pharmacology, Vol. 590, No. 1-3, 2008, pp.327-332. doi:10.1016/j.ejphar.2008.06.007

[23] A. G. Olsson, H. Istad, O. Luurila, S. Stender, J. Tuomilehto, O. Wiklund, H. Southworth, J. Pears and J. W. Wilpshaar, on Behalf of the Rosuvastatin Investigators Group, "Effect of Rosuvastatin and Atorvastatin Compared over 52 Weeks of Treatment in Patients with Hypercholesterolemia," American Heart Journal, Vol. 144, No. 6, 2002, pp. 1044-1051. doi:10.1067/mhj.2002.128049
[24] T. J. Knott, R. J. Pease, L. M. Powell, S. C. Wallis, S. C. Rall Jr., T. L. Inerarity, B. Blackhart, R. Marcel, D. Johnson, M. Filler, A. J. Lusis, B. J. MCarthy, R. W. Mahley, B. Levy-Wilson and J. Scott, "Complete Protein Sequence and Identification of Structural Domains of $\mathrm{Hu}-$ man Apolipoprotein B,” Nature, Vol. 323, No. 6090, 1986, pp. 734-738. doi:10.1038/323734a0

[25] B. A. Griffin and C. J. Packard, "Metabolism of VLDL and LDL Subclasses," Current Opinion in Lipidology, Vol. 5, No. 3, 1994, pp. 200-206. doi:10.1097/00041433-199405030-00007

[26] B. A. Griffin, "Low-Density Lipoprotein Subclasses: Mechanisms of Formation and Modulation," Proceedings of the Nutrition Society, Vol. 56, No. 2, 1997, pp. 693702. doi:10.1079/PNS19970069 